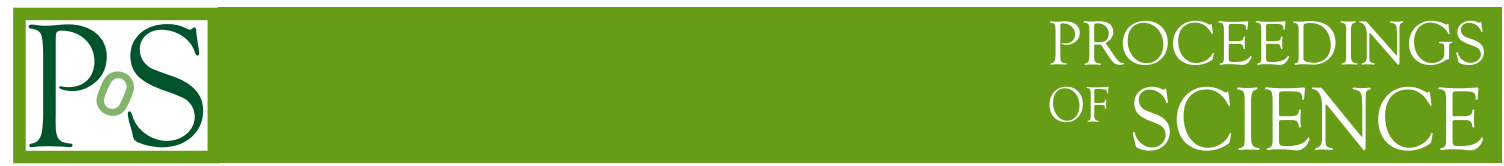

\title{
Hot dust, warm dust and star formation in NLS1s
}

\author{
Rivay Mor* \\ Tel Aviv University \\ E-mail: rivay@wise.tau.ac.il
}

\section{Hagai Netzer}

Tel Aviv University

\begin{abstract}
We present the results of a detailed investigation of the near- to far-infrared continuum emission of a large sample of NLS1s and BLS1s. We fitted the AGN-produced SEDs of 51 NLS1s and 64 BLS1s using a three-component model in order to define the main emission components. Our model consists of: a clumpy torus, dusty narrow-line region clouds, and very hot pure-graphite dust clouds. The AGN SEDs were obtained by subtracting the star formation contribution to the mid-infrared (MIR) emission. The MIR AGN SEDs of NLS1s and BLS1s are found to be very similar regardless of AGN luminosity. We present the covering factors of the dusty components, for all sources, and compare it with the AGN intrinsic properties. We find that the hot dust component is essential to explain the (rest) NIR emission, and that it is consistent with clouds of pure graphite dust composition located between the dust-free broad line region and the "standard" torus. The covering factor of this component is correlated with the AGN bolometric luminosity and anti-correlated with the normalized accretion rate.
\end{abstract}

Narrow-Line Seyfert 1 Galaxies and their place in the Universe April 4-6, 2011

Milano, Italy

\footnotetext{
*Speaker.
} 


\section{Introduction}

According to the unification scheme of active galactic nuclei (AGNs; e.g., Krolik \& Begelman, 1988; Antonucci, 1993) the bulk of the radiation from the central engine is absorbed by an anisotropic, circumnuclear, obscuring structure and re-emitted mainly at mid-infrared (MIR) wavelengths. This structure may be composed of several different physical components. A main component of the obscuring structure is believed to be a dusty torus. The MIR SED of such a torus depends on its dimensions and geometry, the density distribution and the dust grain properties. Other AGN components can contribute to the observed MIR spectrum of AGNs. Some of this emission may originate farther from the central radiation source, at distances exceeding the dimensions of the torus. Dusty clouds in the narrow-line region (NLR) may be the source of such radiation (Schweitzer et al., 2008; Mor et al., 2009, hereafter M09).

Another component of very hot dust at the innermost edge of the torus has been suggested in the past, and in recent years is increasingly being supported by observations. Dust reverberation measurements lead to the conclusion that the near-infrared (NIR) emission in these sources is dominated by thermal radiation from hot dust very close to center (e.g., Minezaki et al., 2004; Suganuma et al., 2006). Several studies fitted the NIR to MIR SEDs of AGNs using a blackbody spectrum to represent emission from hot dust in the inner region of the torus (e.g., Edelson \& Malkan 1986; Barvainis 1987; Kishimoto et al. 2007; Riffel et al. 2009; M09; Deo et al. 2011). More recently Landt et al. (2011) found similar results by fitting the optical to NIR SED of 23 AGNs. The modeled temperature of this component is usually found to be high, $\gtrsim 1200 \mathrm{~K}$, regardless of the AGN luminosity and is consistent with pure-graphite dust emission (M09). Mor \& Trakhtenbrot (2011) used the (observed) optical to MIR SEDs of $~ 15000$ type-I AGNs to conclude that a hot pure-graphite dust component is observed in the vast majority of type-I AGNs.

In these proceedings we present the results of a detailed investigation of a large sample of NLS1s and BLS1s. We fitted the observed NIR to MIR SEDs of 115 type-I AGNs using a 3component model made of a clumpy torus, dusty NLR clouds and very hot pure-graphite dust clouds. These results are discussed in full detail in an upcoming publication by Mor \& Netzer.

\section{Sample Selection and Observations}

Our sample consists of local type-I AGNs collected from several other samples, covering a wide range in luminosity and divided into two groups of NLS1s and BLS1s, according to their FWHM $[\mathrm{H} \beta]$. The sample is based on the one described in Sani et al. (2010) and supplemented with a sample of 26 PG QSOs which is described in detail in Schweitzer et al. (2006), Netzer et al. (2007) and M09. The luminosity range of the entire sample is $L_{5100} \approx 10^{42.2-45.9} \mathrm{erg} \mathrm{s}^{-1}$ where $L_{5100}$ stands for $\lambda L_{\lambda}$ at rest wavelength $5100 \AA$. Spitzer/IRS spectra for all objects were taken in either low or high resolution modes and cover the wavelength range of 5-35 $\mu \mathrm{m}$ in the observed frame. We supplemented the Spitzer spectra with NIR data obtained from the 2MASS extended and point source catalogs (Skrutskie et al., 2006). To account for longer wavelengths of the SED we use IRAS $60 \mu \mathrm{m}$ photometry (Neugebauer et al., 1984).

In order to compute the bolometric luminosity of all sources we adopt the Marconi et al. (2004) "intrinsic", luminosity dependant SED, which provides a polynomial prescription for estimating 
$\lambda L_{\lambda}(4400 \AA)$ at every $L_{\mathrm{bol}}$. We use this prescription for $L_{5100}$ by adopting a $f_{v} \propto v^{-0.5}$ powerlaw approximation. We have estimated the black hole mass $\left(M_{\mathrm{BH}}\right)$ and normalized accretion rate $\left(L / L_{\text {Edd }}\right)$ for all sources using the procedure described in Netzer \& Trakhtenbrot (2007). In this procedure (the "virial" mass determination, see Eq. 1 in Netzer \& Trakhtenbrot 2007), $L_{5100}$ and $\operatorname{FWHM}(\mathrm{H} \beta)$ are combined to obtain $M_{\mathrm{BH}} . L / L_{\mathrm{Edd}}$ is obtained by using the adopted bolometric correction factor.

\section{Spectral Decomposition}

One of the main goals of the present work is to fit the 2-60 $\mu \mathrm{m}$ SED of the sources in our sample, following the general scheme of M09. Our models include different physical components: a very hot graphite-only dust, a clumpy torus with silicate dust, a dusty NLR, and a star forming host galaxy. We start by separating the AGN-related components from the host galaxy component. This subtraction is a major challenge and a major source of uncertainty mainly at longer wavelengths where the emission from star formation is dominant.

We have constructed a library of SF templates using Spitzer/IRS spectra of 5 different star forming galaxies: M82, UGC 2982, NGC 6090, IRAS 22491-1808, and Arp 220. These galaxies span a wide range of IR luminosity $\left(L_{\mathrm{IR}} \approx 10^{10.5-12.2} L_{\odot}\right)$ and PAH luminosities $\left(L_{P A H}(7.7 \mu \mathrm{m}) \approx\right.$ $\left.10^{8.4-9.8} L_{\odot}\right)$. The observed spectra of the templates are smoothed to avoid any systematic features in the subtracted AGN spectra. The ratio between the FIR and MIR fluxes $\left(f_{60} / f_{25}\right)$ in star forming galaxies has a large intrinsic scatter and ranges between about 3 and 13 (e.g., Sanders et al., 2003). For each star forming galaxy we choose 8 values within this range and simulate a $60 \mu \mathrm{m}$ flux accordingly. Finally, our set of SF templates consists of 40 templates covering a large range of IR luminosity, FIR/MIR ratio, and PAH features strengths.

We subtract each template from the observed SEDs using the intensity of the PAH emission features clearly seen in many of our sources. The PAH emission is assumed to be solely due to the $\mathrm{SF}$ in the host galaxy. For each SF template we choose a normalization factor such that after the subtraction, the observed PAH features are completely gone from the spectrum, and the remaining flux at the relevant wavelength range is consistent with the level of noise. For objects with no PAH detection the subtraction is limited according to the level of noise in the relevant wavelength ranges in their spectrum.

The first AGN-related component represents a dusty torus surrounding the central energy source. We use the clumpy torus models of Nenkova et al. (2008a) in a similar way to that described in M09. Unfortunately the normalizations of the models (those calculated before September 2010 and used in M09) were found to be off by a factor of $\sim 2-3$ (see erratum by Nenkova et al., 2010). The corrected clumpy torus models are very useful to determine the contribution of the torus component to the IR SED (i.e. the torus covering factor). The first parameter of the clumpy torus model is the inner radius of the cloud distribution that is set to the dust sublimation radius $R_{d, S i}$. This corresponds to a dust sublimation temperature $T_{\text {sub }}$ that depends on the grain properties and mixture. All models considered here assume spherical dust grains with MRN size distribution (Mathis et al., 1977). The dust has a standard galactic mix of 53\% silicates and $47 \%$ graphite. The optical properties of graphite are taken from Draine (2003) and for silicates from Ossenkopf et al. (1992, OHM). Nenkova et al. (2008b) adopted a sublimation radius appropriate for an "average" 
grain, representing the properties above and a sublimation temperature of $1500 \mathrm{~K}$. This is given by

$$
R_{d, S i} \simeq 1.3 \times\left(\frac{L_{\mathrm{bol}}}{10^{46} \mathrm{erg} \mathrm{s}^{-1}}\right)^{1 / 2}\left(\frac{1500 \mathrm{~K}}{T_{\text {sub }}}\right)^{2.6} \mathrm{pc} .
$$

The second component of the model represents a collection of dusty NLR clouds. The motivation for this component is explained in Schweitzer et al. (2008) and M09 where it was shown that such a component can contribute significantly to the MIR flux of luminous AGNs. The properties assumed here for these clouds are similar to the ones used in M09. We assume constant column density clouds with $N_{\mathrm{H}}=10^{21.5} \mathrm{~cm}^{-2}$. We further assume constant hydrogen density of $10^{5} \mathrm{~cm}^{-3}$, solar composition and galactic dust-to-gas ratio. The important physical parameters for this component are the distance from the central source to the clouds (which determines the dust temperature), the incident SED, and the dust column density.

The third component represents a collection of dusty clouds of gas located at the inner edge of the torus and with a pure graphite dust composition. The motivation for this component is explained in M09 where it was shown that such a component is necessary to explain the NIR emission of typeI QSOs. Mor \& Trakhtenbrot (2011) have shown that a hot dust component is present in $\gtrsim 80 \%$ of type-I AGNs and is significantly luminous. The pure-graphite dust must be external to the broad line region (BLR), where dust cannot survive, and internal to the "standard" clumpy torus, where the distances are large enough to allow silicate-type dust. Here we suggest that the pure-graphite dust is located in BLR clouds that are between the silicate and graphite sublimation radii. The pure-graphite dust sublimation radius is given by

$$
R_{d, C} \simeq 0.5 \times\left(\frac{L_{\mathrm{bol}}}{10^{46} \mathrm{erg} \mathrm{s}^{-1}}\right)^{1 / 2}\left(\frac{1800 \mathrm{~K}}{T_{\mathrm{sub}}}\right)^{2.8} \mathrm{pc} .
$$

The fitting procedure starts with the SF template subtracted SED that is assumed to represent only the AGN-produced continuum. Although the SF component is subtracted prior to the model fitting, it introduces another degree of freedom to the procedure. To fit the SED with a 3-component model we use $\chi^{2}$ minimization in a similar way to that described in M09. The only difference is that the normalization of the torus component is now a free parameter. Fig. 1 shows the best fit model for a representative case, the NLS1 Mrk 896.

\section{Intrinsic AGN SED}

The subtracted spectra are used for constructing a median intrinsic AGN SEDs for different groups in the sample divided by luminosity and by emission line width. For all the spectra in each group we calculate the median luminosity in every wavelength bin, to get a median intrinsic AGN SED. The right panel of Figure 2 shows the median AGN SED for the two populations of narrowand broad-line AGNs in our sample. The left panel shows the median AGN SED for different luminosity groups in our sample. Examining Figure 2 it is evident that the general shape of the SEDs is very similar regardless of luminosity or line widths. All SEDs exhibit three distinct peaks at short $(\lesssim 5 \mu \mathrm{m})$ and medium $(\sim 10$ and $\sim 20 \mu \mathrm{m})$ wavelengths. The last two peaks are likely dominated by silicate dust, corresponding to the 10 and $18 \mu \mathrm{m}$ broad emission features. 

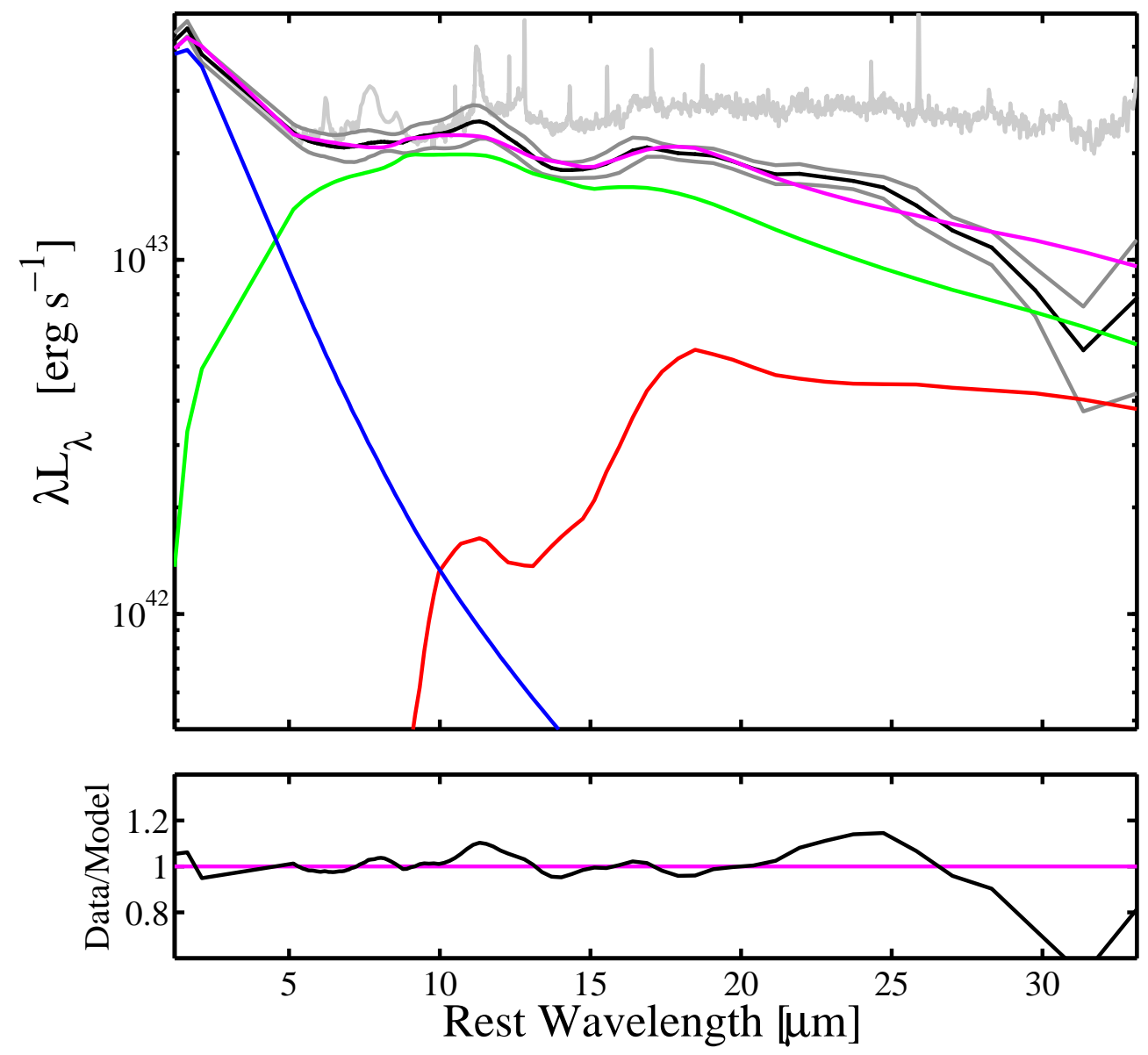

Figure 1: Best fit using the three-component model for a representative case, the NLS1 Mrk 896. Top panel shows the best fit model (magenta), the observed (gray) and the subtracted and binned (black) spectra. We also show individual components: torus (green), NLR (red) and hot pure-graphite dust (blue). In the bottom panel we show the quality of the fit in each wavelength bin, by calculating the ratio between the model and the fitted data.

Our result is consistent with the SEDs found by Netzer et al. (2007) for the QUEST sample. The QUEST sample represents the high end of the AGN luminosity range of our current sample, and mostly consists of broad line sources. Netzer et al. (2007) also divided the QUEST sample into two groups of sources with strong and weak FIR emission. Again, the shapes of the AGN SEDs were remarkably similar. Deo et al. (2009) employed a similar subtraction method to a large sample of type-I and -II Seyfert galaxies. These authors find that the AGN continuum emission drops rapidly beyond $\sim 20 \mu \mathrm{m}$ for all AGN types, regardless of SF activity. Recently, Mullaney et al. (2011) employed a slightly different method to determine the intrinsic AGN SED of a sample of type-I and type-II AGNs. These authors fit the IR SEDs, simultaneously, using a SF template and a broken power law that represents the AGN emission. They find that the warm dust peak is located at somewhat longer wavelengths (between $\sim 15$ and $45 \mu \mathrm{m}$ ) before dropping rapidly towards FIR 

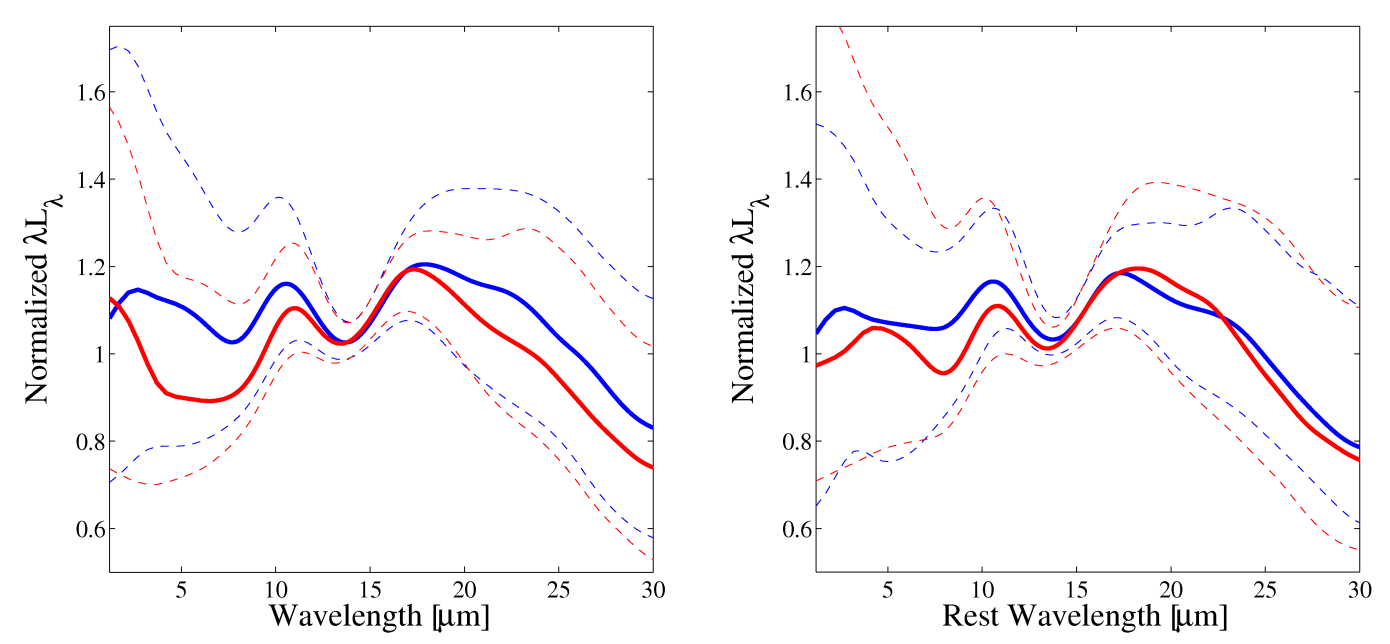

Figure 2: Intrinsic AGN SEDs. Right: median subtracted SEDs for two sub-groups of NLS1s (blue) and BLS1s (red). Dashed lines represent the 25th and 75th percentiles of each sub-group. Left: median subtracted SEDs for two sub-groups divided according to their luminosity at $6 \mu \mathrm{m}$. The blue and red curves represent sources above and below the median luminosity at $6 \mu \mathrm{m}\left(10^{44.2} \mathrm{erg} \mathrm{s}^{-1}\right)$, respectively. As in the right panel, dashed lines represent the 25 th and 75 th percentiles of each sub-group. All SEDs have very similar shape and exhibit three peaks at short $(\lesssim 5 \mu \mathrm{m})$ and medium $(\sim 10$ and $\sim 20 \mu \mathrm{m})$ wavelengths.

wavelengths. This result is not confirmed by our analysis, probably due to the different way of accounting for the SF contribution and the different SF templates used. We suspect that it may also be affected by the presence of type-II AGNs in their sample and the difficulty to properly account for the host contribution in these sources.

\section{Hot Pure-Graphite Dust}

A major goal of our work is to explain the NIR emission in type-I AGNs and to identify the physical properties of the component responsible for this emission. Here we suggest that the emission is due to hot dust clouds with pure graphite dust composition. The most important parameter of the hot dust component, determined by the fitting procedure, is its luminosity $\left(L_{\mathrm{HD}}\right)$. Since the hot dust is optically thick at all UV-optical wavelengths, $L_{\mathrm{HD}}$ is a measure of the covering factor of this component $\left(\mathrm{CF}_{\mathrm{HD}}\right)$. Figure 3 shows a strong correlation between $L_{\mathrm{HD}}$ and $L_{\mathrm{bol}}$. NLS1s (blue circles) exhibit a similar trend to that of BLS1s (red circles) although the scatter is larger. Fig. 3 also show the results of Mor \& Trakhtenbrot (2011) (gray dots). These authors fitted the (observed) optical to MIR SEDs of $\sim 15000$ high luminosity QSOs at $0.75<\mathrm{z}<2$, and measured the luminosity of the hot dust component. The relation between $L_{\mathrm{HD}}$ and $L_{\mathrm{bol}}$, over a large luminosity range, indicates that $\mathrm{CF}_{\mathrm{HD}}$ in type-I AGNs spans a relatively limited range $(\sim 0.1-0.2$, see below). The NLS1s that lie much below the relation of Fig. 3 may represent sources with exceptionally low $\mathrm{CF}_{\mathrm{HD}}$ (see below).

A major assumption of this work is that the entire MIR spectrum, after starburst subtraction, is reprocessed AGN radiation. This can be used to deduce the covering factor of the central source by the three components (see also M09 and references therein). The covering factors are defined by 


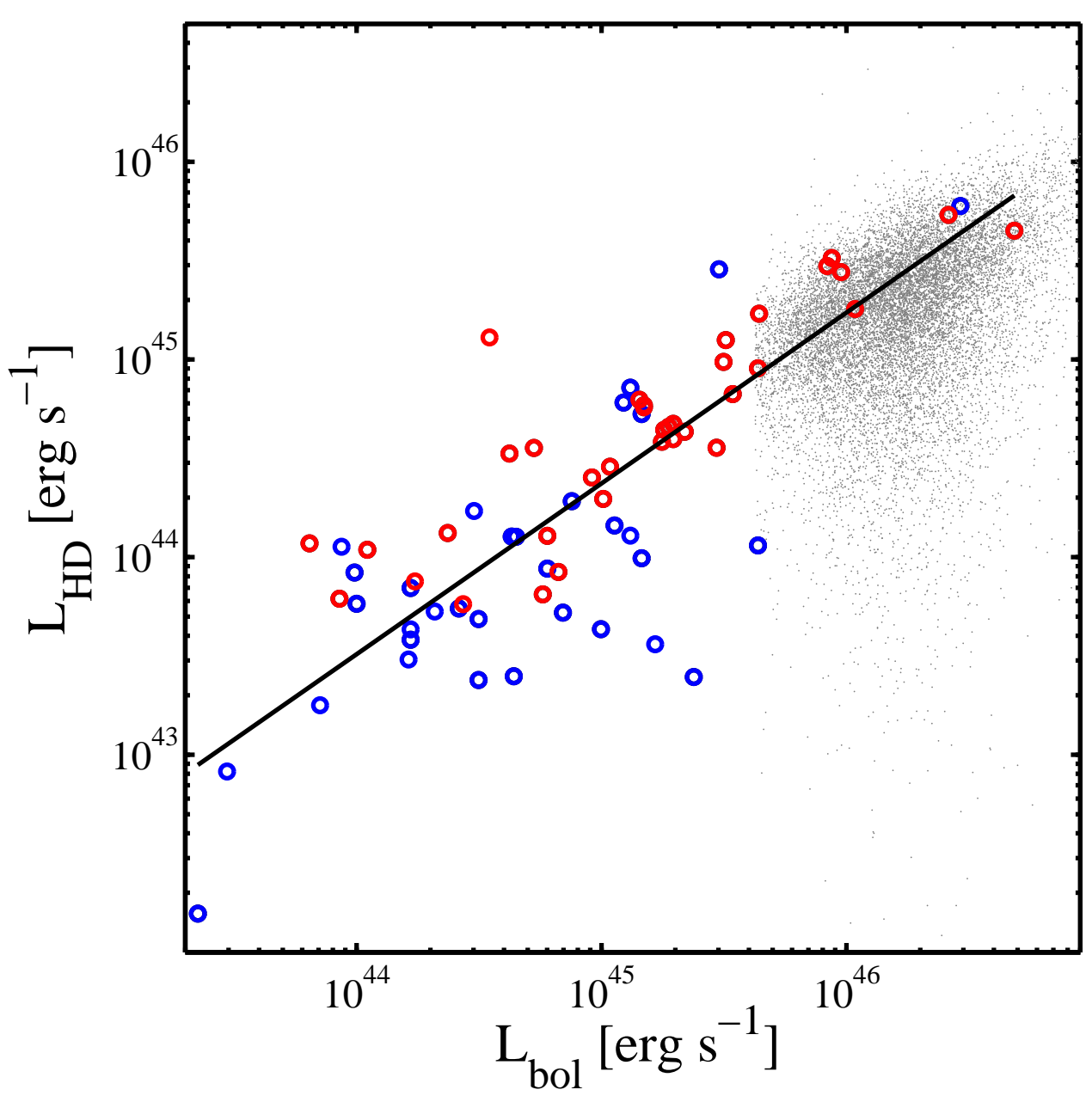

Figure 3: Luminosity of the hot dust component vs. AGN bolometric luminosity. Results from the current work are shown as blue and red circles (NLS1s and BLS1s, respectively), Results for a much larger sample of high luminosity QSOs from Mor \& Trakhtenbrot (2011) are shown in gray. The black line represents an OLS fit to the data of the current work.

the ratio between the total luminosity of each component and $L_{\mathrm{bol}}$. Figure 4 shows the cumulative distribution functions of the covering factors of the different components. In all panels the blue lines represents the NLS1s in the sample and the red lines represents the BLS1s. As can be seen in Fig. 4, NLS1s tend to have lower covering factors compared with BLS1s. The median value of $\mathrm{CF}_{\mathrm{HD}}$ for the NLS1s is 0.23 and for the BLS1s 0.27 . The median value of the torus covering factor is 0.24 for the NLS1s and 0.33 for the BLS1s. The covering factor of the NLR component has a median value of 0.03 for the NLS1s and 0.07 for the BLS1s.

Sani et al. (2010) found that NLS1s tend to have stronger SF activity compared with BLS1s at a given AGN luminosity. Since we fit the SF subtracted SED this may effect the derived torus and NLR covering factors. However, this cannot explain the difference in the covering factors of 


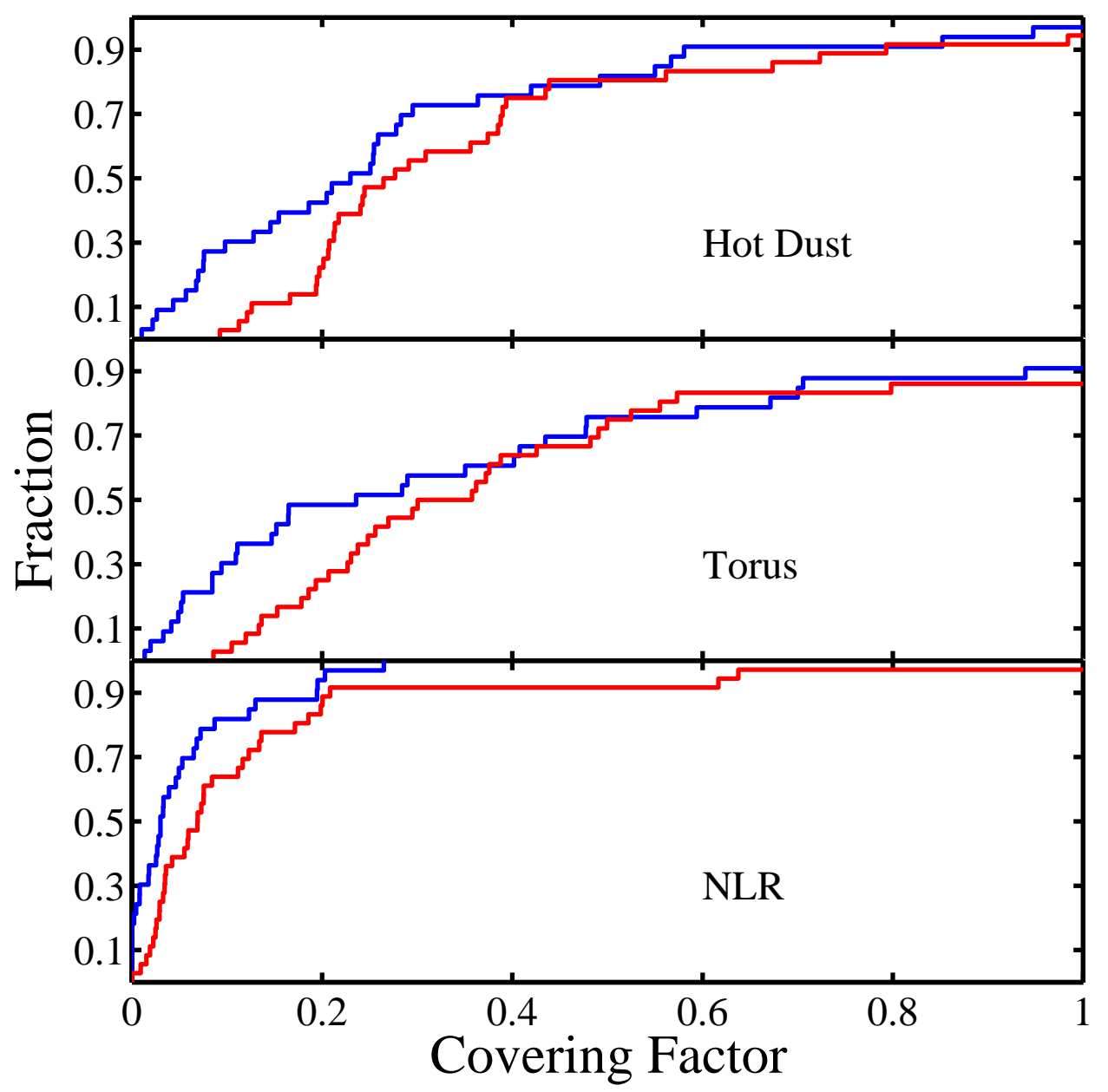

Figure 4: Cumulative distribution functions of the covering factors of the three dusty components. For all components, the covering factors tend to be smaller in NLS1s (blue) than in BLS1(red). The distribution of $\mathrm{CF}_{\mathrm{HD}}$ and the torus covering factor are very similar, indicating that about half of the obscuration is due to hot pure-graphite dust clouds which are not included in "standard" torus models.

the hot dust component since the SF contribution to the 1-5 $\mu \mathrm{m}$ wavelength range is very small. Host contribution to the optical range (i.e. to $L_{5100}$ ) would result in an overestimation of the AGN bolometric luminosity and consequently lower the estimated covering factor. Such host contribution to the optical spectrum is, however, more significant in lower luminosity AGNs regardless of their line widths.

In order to explore the relation with $\mathrm{AGN}$ properties we focus on $\mathrm{CF}_{\mathrm{HD}}$ and compare it against $L_{\mathrm{bol}}, M_{\mathrm{BH}}$, and $L / L_{\mathrm{Edd}}$. Fig. 5 shows $\mathrm{CF}_{\mathrm{HD}}$ against these properties for the current sample of NLS1s (blue circles) and BLS1s (red circles). The results of Mor \& Trakhtenbrot (2011, gray dots) are shown for comparison. The black dashed lines in Fig. 5 represent the 99th percentiles boundaries of the $\mathrm{CF}_{\mathrm{HD}}$ distribution of the Mor \& Trakhtenbrot (2011) sample. The percentiles were calculated by assuming that the $\mathrm{CF}_{\mathrm{HD}}$ distribution, in any a certain luminosity bin, should be symmetrical 
around the peak value and by mirroring the high- $\mathrm{CF}_{\mathrm{HD}}$ side of the distribution. This is done in each (0.2 dex) luminosity bin separately. Mor \& Trakhtenbrot (2011) suggest that all the points which lie below the lower dashed lines can be regarded as hot dust poor AGNs. The fraction of such sources in their sample is $\sim 20 \%$ and does not depend on source luminosity.

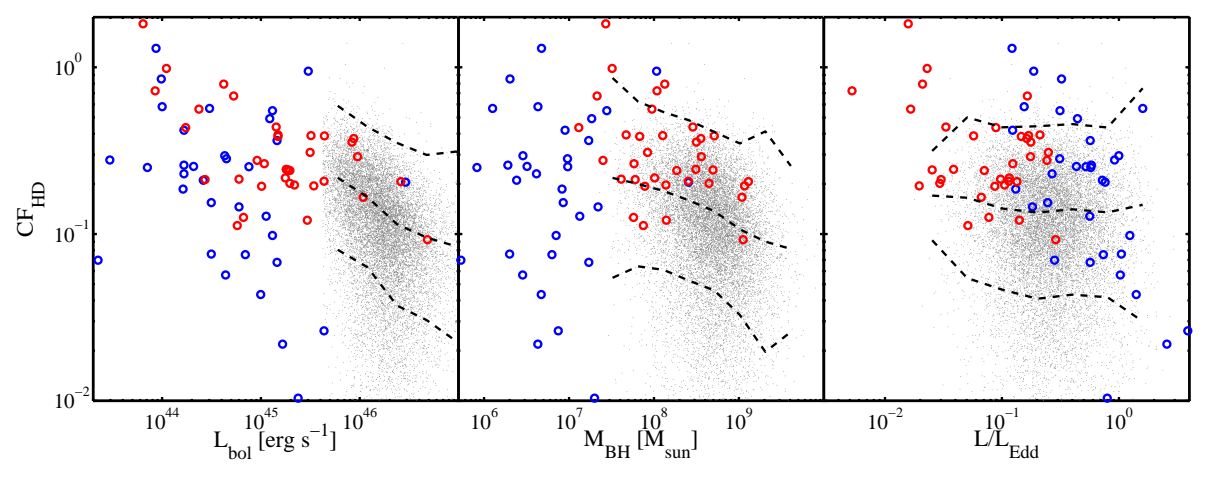

Figure 5: Covering factor of the hot pure-graphite dust component against $L_{\mathrm{bol}}$ (left), $M_{\mathrm{BH}}$ (middle), and $L / L_{\text {Edd }}$ (left panel). In all panels, NLS1s are represented by the blue circles and BLS1s in red. Results of Mor \& Trakhtenbrot (2011, gray dots) are shown for comparison. Dashed black lines represent the peak of the $\mathrm{CF}_{\mathrm{HD}}$ distribution and the $3 \sigma$-equivalent range - see text

The NLS1s in our sample have lower $M_{\mathrm{BH}}$ than the BLS1s. As can be seen in the middle panel of Fig. 5, most of the sources with $\log \left(M_{\mathrm{BH}} / M_{\odot}\right)<7.3$ are NLS1s and $\sim 20 \%$ of them have $\mathrm{CF}_{\mathrm{HD}}$ lower than 0.1. No significant correlation was found between $\mathrm{CF}_{\mathrm{HD}}$ and $M_{\mathrm{BH}}$. If the NLS1s that have very low $\mathrm{CF}_{\mathrm{HD}}$ are truly hot dust poor AGNs it may suggest that these sources are more common in low $M_{\mathrm{BH}}$ sources.

$L / L_{\mathrm{Edd}}$ is known to be higher in NLS1s. The right panel of Fig. 5 shows $\mathrm{CF}_{\mathrm{HD}}$ against $L / L_{\mathrm{Edd}}$. There is a clear anti-correlation over two orders of magnitude in $L / L_{\text {Edd }}$. The NLS1s that lie below the $\mathrm{CF}_{\mathrm{HD}}-L_{\mathrm{bol}}$ and $\mathrm{CF}_{\mathrm{HD}}-M_{\mathrm{BH}}$ relations are also the sources with the highest $L / L_{\mathrm{Edd}}$. Mor \& Trakhtenbrot (2011) did not find any significant correlation with $L / L_{\text {Edd }}$. However, their sample spans a narrow range of $L / L_{\text {Edd }}$.

The second parameter that is determined by the fitting procedure is the distance from the center to the hot dust component $\left(R_{\mathrm{HD}}\right)$. Figure 6 shows this distance against $L_{\mathrm{bol}}$. The silicate and pure-graphite dust sublimation radii defined by equations 3.1 and 3.2, respectively, are also shown. We also show the relation between the emissivity weighted BLR radius for the $\mathrm{H} \beta$ line, $R_{\mathrm{BLR}}$ and $L_{\mathrm{bol}}$. This distance is based on an up-to-date version of the Kaspi et al. (2005) relation taking into account the modifications of Bentz et al. (2009): $R_{\mathrm{BLR}}=0.02 \times\left(L_{5100} / 10^{44}\right)^{0.62}[p c]$. Fig. 6 demonstrates that clouds are clearly situated outside the dust-free BLR and within the edge of the "standard" silicate-dust torus. 


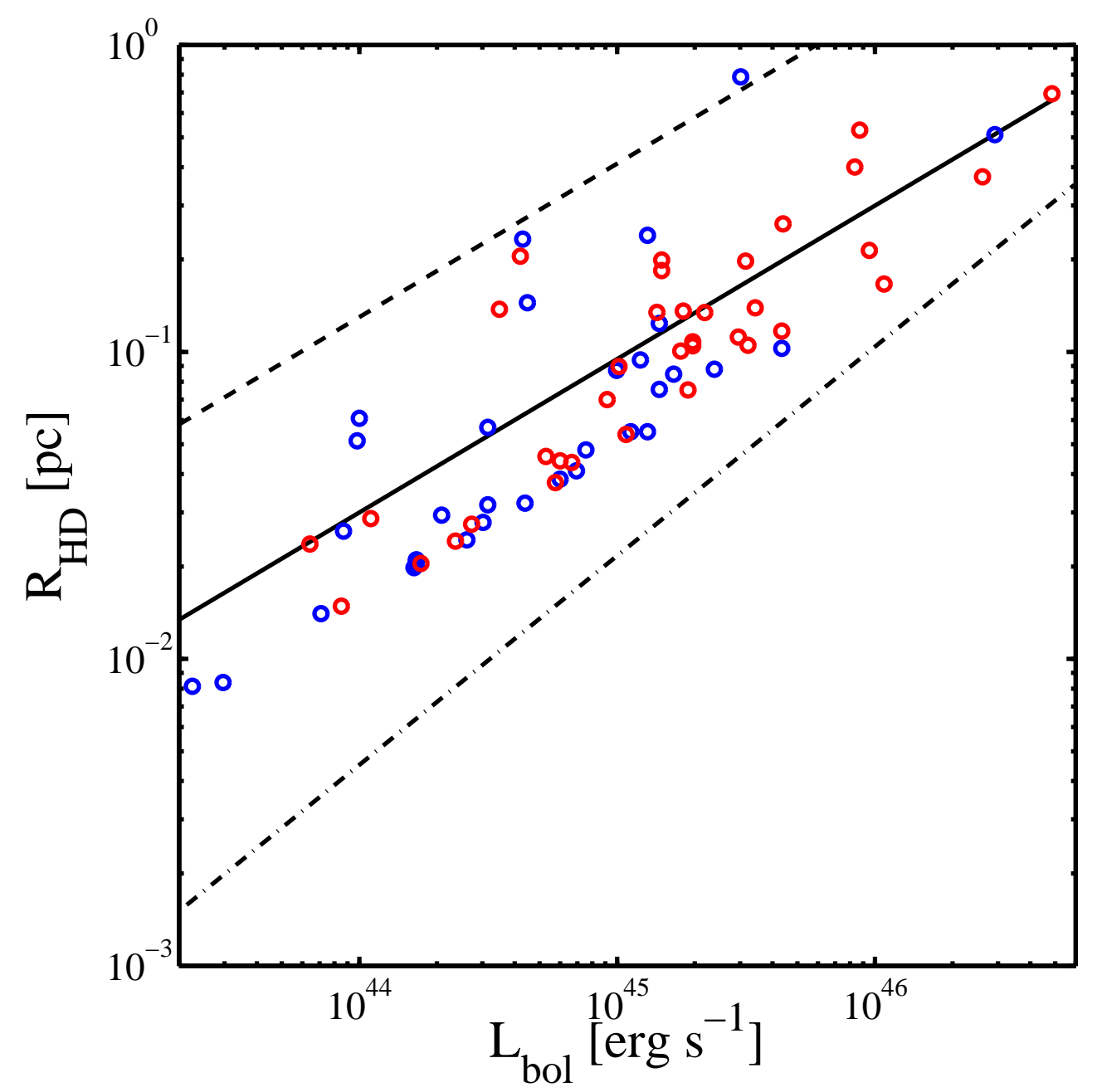

Figure 6: Distance to the hot pure-graphite dust components vs. AGN bolometric luminosity for two subgroups of NLS1s (blue) and BLS1s (red). Dashed and solid black lines represent the sublimation radii of silicate and pure-graphite dust, respectively. Dot-dashed line represent the $R_{\mathrm{BLR}}-L_{\mathrm{bol}}$ relation (see text).

\section{References}

Antonucci, R. 1993, Annual Review of Astronomy and Astrophysics, 31, 473

Barvainis, R. 1987, The Astrophysical Journal, 320, 537

Bentz, M. C., Peterson, B. M., Netzer, H., Pogge, R. W., \& Vestergaard, M. 2009, The Astrophysical Journal, 697, 160

Deo, R. P., Richards, G. T., Crenshaw, D. M., \& Kraemer, S. B. 2009, The Astrophysical Journal, 705,14

Deo, R. P., Richards, G. T., Nikutta, R., Elitzur, M., Gallagher, S. C., Ivezić, v., \& Hines, D. 2011, The Astrophysical Journal, 729, 108 
Draine, B. T. 2003, The Astrophysical Journal, 598, 1017

Edelson, R. A., \& Malkan, M. A. 1986, The Astrophysical Journal, 308, 59

Kaspi, S., Maoz, D., Netzer, H., Peterson, B. M., Vestergaard, M., \& Jannuzi, B. T. 2005, The Astrophysical Journal, 629, 61

Kishimoto, M., Hönig, S. F., Beckert, T., \& Weigelt, G. 2007, Astronomy and Astrophysics, 476, 713

Krolik, J. H., \& Begelman, M. C. 1988, The Astrophysical Journal, 329, 702

Landt, H., Elvis, M., Ward, M. J., Bentz, M. C., Korista, K. T., \& Karovska, M. 2011, Monthly Notices of the Royal Astronomical Society, 414, 218

Marconi, A., Risaliti, G., Gilli, R., Hunt, L. K., Maiolino, R., \& Salvati, M. 2004, Monthly Notices of the Royal Astronomical Society, 351, 169

Mathis, J. S., Rumpl, W., \& Nordsieck, K. H. 1977, The Astrophysical Journal, 217, 425

Minezaki, T., Yoshii, Y., Kobayashi, Y., Enya, K., Suganuma, M., Tomita, H., Aoki, T., \& Peterson, B. A. 2004, The Astrophysical Journal, 600, L35

Mor, R., Netzer, H., \& Elitzur, M. 2009, The Astrophysical Journal, 705, 298

Mor, R., \& Trakhtenbrot, B. 2011, eprint arXiv:1105.3198

Mullaney, J. R., Alexander, D. M., Goulding, A. D., \& Hickox, R. C. 2011, eprint arXiv: 1102.1425

Nenkova, M., Sirocky, M. M., Ivezić, v., \& Elitzur, M. 2008a, The Astrophysical Journal, 685, 147

Nenkova, M., Sirocky, M. M., Nikutta, R., Ivezić, v., \& Elitzur, M. 2008b, The Astrophysical Journal, 685, 160

—. 2010, The Astrophysical Journal, 723, 1827

Netzer, H., et al. 2007, The Astrophysical Journal, 666, 806

Neugebauer, G., et al. 1984, The Astrophysical Journal, 278, L1

Ossenkopf, V., Henning, T., \& Mathis, J. S. 1992, Astronomy and Astrophysics (ISSN 0004-6361), 261,567

Riffel, R. A., Storchi-Bergmann, T., Dors, O. L., \& Winge, C. 2009, Monthly Notices of the Royal Astronomical Society, 393, 783

Sanders, D. B., Mazzarella, J. M., Kim, D.-C., Surace, J. A., \& Soifer, B. T. 2003, The Astronomical Journal, 126, 1607

Sani, E., Lutz, D., Risaliti, G., Netzer, H., Gallo, L. C., Trakhtenbrot, B., Sturm, E., \& Boller, T. 2010, Monthly Notices of the Royal Astronomical Society, 403, 1246 
Schweitzer, M., et al. 2006, The Astrophysical Journal, 649, 79

—. 2008, The Astrophysical Journal, 679, 101

Skrutskie, M. F., et al. 2006, The Astronomical Journal, 131, 1163

Suganuma, M., et al. 2006, The Astrophysical Journal, 639, 46 